\title{
Criteria for a certain class of the Carathéodory functions and their applications
}

Nak Eun Cho ${ }^{1}$, H.M. Srivastava ${ }^{2,3,4}$, Ebrahim Analouei Adegani ${ }^{5 *}$ and Ahmad Motamednezhad ${ }^{5}$

\section{"Correspondence:}

analoey.ebrahim@gmail.com

${ }^{5}$ Faculty of Mathematical Sciences,

Shahrood University of Technology,

Shahrood, Iran

Full list of author information is

available at the end of the article

\begin{abstract}
In this paper, we obtain some potentially useful conditions (or criteria) for the Carathéodory functions as a certain class of analytic functions by applying Nunokawa's lemma. We also obtain several conditions for strong starlikeness and close-to-convexity as special cases of the main results presented here.
\end{abstract}

MSC: Primary 30C45; secondary 30C80

Keywords: Carathéodory functions; Differential subordination; Strongly close-to-convex functions; Starlike functions; Strongly starlike functions

\section{Introduction and preliminaries}

Let $\mathcal{A}$ be a class of functions $f$ of the following normalized form:

$$
f(z)=z+\sum_{n=2}^{\infty} a_{n} z^{n}
$$

which are analytic in the open unit disk $\mathbb{U}$ given by

$$
\mathbb{U}:=\{z: z \in \mathbb{C} \text { and }|z|<1\} .
$$

Let $\widetilde{\mathcal{P}}(\alpha)$ be a class of functions $p$ of the form

$$
p(z)=\sum_{n=0}^{\infty} p_{n} z^{n}
$$

which are analytic in $\mathbb{U}$ with $p(0)=1$ and

$$
|\arg (p(z))|<\frac{\alpha \pi}{2} \quad(z \in \mathbb{U} ; 0<\alpha \leqq 1) .
$$

Then, in the special case when $\alpha=1, \widetilde{\mathcal{P}}(1)$ is the well-known class of Carathéodory functions in $\mathbb{U}$ (see [8] and [9]; see also the recent developments on this subject in, for example, $[19,20,23]$, and [28]).

(c) The Author(s) 2020. This article is licensed under a Creative Commons Attribution 4.0 International License, which permits use, sharing, adaptation, distribution and reproduction in any medium or format, as long as you give appropriate credit to the original author(s) and the source, provide a link to the Creative Commons licence, and indicate if changes were made. The images or other third party material in this article are included in the article's Creative Commons licence, unless indicated otherwise in a credit line to the material. If material is not included in the article's Creative Commons licence and your intended use is not permitted by statutory regulation or exceeds the permitted use, you will need to obtain permission directly from the copyright holder. To view a copy of this licence, visit http://creativecommons.org/licenses/by/4.0/. 
For two functions $f$ and $F$, which are analytic in $\mathbb{U}$, we say that the function $f$ is subordinate to the function $F$ in $\mathbb{U}$ and we write $f(z) \prec F(z)$ if there exists a Schwarz function $\omega$, which is analytic in $\mathbb{U}$ with

$$
\omega(0)=0 \quad \text { and } \quad|\omega(z)|<1 \quad(z \in \mathbb{U})
$$

such that $f(z)=F(\omega(z))$ for all $z \in \mathbb{U}$. In particular, if the function $F$ is univalent in $\mathbb{U}$, then we have the following equivalence:

$$
f(z) \prec F(z) \quad \Longleftrightarrow \quad f(0)=F(0) \quad \text { and } \quad f(\mathbb{U}) \subset F(\mathbb{U})
$$

Several recent investigations on various applications of differential subordination and differential superordination were reported in, for example, [21, 22, 26, 27] (see also [4, 5], and [6]).

We denote by $\widetilde{\mathcal{S}}^{*}(\alpha)$ the subclass of $\mathcal{A}$ consisting of functions which are strongly starlike of order $\alpha$ in $\mathbb{U}$, that is,

$$
\widetilde{\mathcal{S}}^{*}(\alpha):=\left\{f: f \in \mathcal{A} \text { and }\left|\arg \left(\frac{z f^{\prime}(z)}{f(z)}\right)\right|<\frac{\alpha \pi}{2}(z \in \mathbb{U} ; 0<\alpha \leqq 1)\right\}
$$

Thus, in particular, $\mathcal{S}^{*}:=\widetilde{\mathcal{S}}^{*}(1)$ is the class of starlike functions in the open unit disk $\mathbb{U}$.

By means of the principle of subordination between analytic functions, the above definition is equivalent to

$$
\widetilde{\mathcal{S}}^{*}(\alpha):=\left\{f: f \in \mathcal{A} \text { and } \frac{z f^{\prime}(z)}{f(z)} \prec\left(\frac{1+z}{1-z}\right)^{\alpha}(z \in \mathbb{U} ; 0<\alpha \leqq 1)\right\} .
$$

We also denote by $\widetilde{\mathcal{C C}}(\alpha)$ the subclass of $\mathcal{A}$ consisting of functions that are strongly closeto-convex of order $\alpha$ in $\mathbb{U}$ if there exists a function $g \in \mathcal{S}^{*}$ such that

$$
\left|\arg \left(\frac{z f^{\prime}(z)}{g(z)}\right)\right|<\frac{\alpha \pi}{2} \quad(z \in \mathbb{U} ; 0 \leqq \alpha<1) .
$$

In particular, $\mathcal{C C}:=\widetilde{\mathcal{C C}}(1)$ is the class of close-to-convex functions in the open unit disk $\mathbb{U}$. Furthermore, we denote by $\widetilde{\mathcal{C}}(\alpha)$ the subclass of $\mathcal{A}$ consisting of functions satisfying the following condition:

$$
\left|\arg \left(f^{\prime}(z)\right)\right|<\frac{\alpha \pi}{2} \quad(z \in \mathbb{U} ; 0 \leqq \alpha<1) .
$$

In particular, $\mathcal{C}:=\widetilde{\mathcal{C}}(1)$ is a subclass of close-to-convex functions in the open unit disk $\mathbb{U}$.

In the year 1978, Miller and Mocanu [14] introduced the method of differential subordinations. Then, in recent years, several authors have obtained several applications of the method of differential subordinations in geometric function theory by using differential subordination associated with starlikeness, convexity, close-to-convexity, and so on (see, for example, $[1-3,7,10-13,17,18,24,25])$. The object of the present paper is to derive various potentially useful conditions (or criteria) for the Carathéodory functions as a certain class of analytic functions in the open unit disk $\mathbb{U}$ by using a lemma given by 
Nunokawa (see [15] and [16]). Further, we give some applications to strong starlikeness and close-to-convexity.

The following lemma will be used in proving our main result.

Lemma 1.1 (see [15] and [16]) Let the function $p(z)$ given by

$$
p(z)=1+\sum_{n=m}^{\infty} c_{n} z^{n} \quad\left(c_{m} \neq 0\right)
$$

be analytic in $\mathbb{U}$ with

$$
p(0)=1 \quad \text { and } \quad p(z) \neq 0 \quad(z \in \mathbb{U}) .
$$

If there exists a point $z_{0}\left(\right.$ with $\left.\left|z_{0}\right|<1\right)$ such that

$$
|\arg (p(z))|<\frac{\beta \pi}{2} \quad\left(|z|<\left|z_{0}\right|\right)
$$

and

$$
\left|\arg \left(p\left(z_{0}\right)\right)\right|=\frac{\beta \pi}{2}
$$

for some $\beta>0$, then

$$
\frac{z_{0} p^{\prime}\left(z_{0}\right)}{p\left(z_{0}\right)}=i k \beta \quad(i=\sqrt{-1}),
$$

where

$$
k \geqq \frac{m\left(a+a^{-1}\right)}{2} \geqq m \quad \text { when } \arg \left(p\left(z_{0}\right)\right)=\frac{\beta \pi}{2}
$$

and

$$
k \leqq-\frac{m\left(a+a^{-1}\right)}{2} \leq-m \quad \text { when } \arg \left(p\left(z_{0}\right)\right)=-\frac{\beta \pi}{2},
$$

where

$$
\left[p\left(z_{0}\right)\right]^{1 / \beta}= \pm i a \text { and } a>0 .
$$

\section{Sufficient conditions for strong starlikeness and close-to-convexity}

Theorem 2.1 Let $p$ be an analytic function in $\mathbb{U}$, with $p(0)=1, p^{\prime}(0) \neq 0$, and $p(z) \neq 0$ for $z \in \mathbb{U}$, that satisfies the following inequality:

$$
\left|[p(z)]^{2}+\frac{z p^{\prime}(z)}{p(z)}\right|<A(\alpha)|p(z)|
$$

where

$$
A(\alpha)= \begin{cases}\sqrt{\frac{\alpha^{2}}{4}\left[\left(\frac{1+\alpha}{1-\alpha}\right)^{(1-\alpha) / 2}+\left(\frac{1+\alpha}{1-\alpha}\right)^{(-1-\alpha) / 2}\right]^{2}+2 \alpha \sin (\pi \alpha)} & \text { if } 0<\alpha<1, \\ \frac{1}{2} & \text { if } \alpha=1 .\end{cases}
$$

Then $p \in \widetilde{\mathcal{P}}(\alpha)$. 
Proof To prove the result asserted by Theorem 2.1, we suppose that there exists a point $z_{0} \in \mathbb{U}$ such that

$$
|\arg (p(z))|<\frac{\alpha \pi}{2} \quad \text { for }|z|<\left|z_{0}\right|
$$

and

$$
\left|\arg \left(p\left(z_{0}\right)\right)\right|=\frac{\alpha \pi}{2}
$$

Then, from Lemma 1.1, it follows that

$$
\frac{z p^{\prime}\left(z_{0}\right)}{p\left(z_{0}\right)}=i k \alpha
$$

where $\left[p\left(z_{0}\right)\right]^{\frac{1}{\alpha}}= \pm i a(a>0)$ and $k$ is given by (1.2) or (1.3) for $m=1$.

For the case when

$$
\left[p\left(z_{0}\right)\right]^{\frac{1}{\alpha}}=i a \quad(a>0)
$$

we have

$$
\begin{aligned}
\left.\mid] p\left(z_{0}\right)\right]^{2}+\frac{z p^{\prime}\left(z_{0}\right)}{p\left(z_{0}\right)} \mid & =\left|p\left(z_{0}\right)\right|\left|p\left(z_{0}\right)+\frac{z p^{\prime}\left(z_{0}\right)}{p\left(z_{0}\right)} \frac{1}{p\left(z_{0}\right)}\right|=\left|p\left(z_{0}\right)\right|\left|(i a)^{\alpha}+i k \alpha \frac{1}{(i a)^{\alpha}}\right| \\
& =\left|p\left(z_{0}\right)\right|\left|a^{\alpha} e^{i \pi \alpha / 2}+\frac{k \alpha}{a^{\alpha}} e^{i \pi(1-\alpha) / 2}\right| .
\end{aligned}
$$

From (2.3) for $\alpha=1$, we find that

$$
\begin{aligned}
\left|\left[p\left(z_{0}\right)\right]^{2}+\frac{z p^{\prime}\left(z_{0}\right)}{p\left(z_{0}\right)}\right| & =\left|p\left(z_{0}\right)\right|\left|a^{\alpha} e^{i \pi \alpha / 2}+\frac{k \alpha}{a^{\alpha}} e^{i \pi(1-\alpha) / 2}\right|=\left|p\left(z_{0}\right)\right|\left|a e^{i \pi / 2}+\frac{k}{a}\right| \\
& =\left|p\left(z_{0}\right)\right|\left|i a+\frac{k}{a}\right|=\left|p\left(z_{0}\right)\right| \sqrt{a^{2}+\left(\frac{k}{a}\right)^{2}}\left(\frac{k}{a} \geqq \frac{a^{2}+1}{2 a^{2}} \geqq \frac{1}{2}\right) \\
& \geqq\left|p\left(z_{0}\right)\right| \sqrt{\frac{1}{4}}=\left|p\left(z_{0}\right)\right| \frac{1}{2} .
\end{aligned}
$$

Also, since

$$
\frac{k \alpha}{a^{\alpha}} \geqq \frac{\alpha}{2}\left(a^{1-\alpha}+a^{-1-\alpha}\right),
$$

by applying (2.3) for $0<\alpha<1$ with

$$
k \geqq \frac{\left(a+a^{-1}\right)}{2} \geqq 1,
$$

we deduce that

$$
\left|\left[p\left(z_{0}\right)\right]^{2}+\frac{z p^{\prime}\left(z_{0}\right)}{p\left(z_{0}\right)}\right|=\left|p\left(z_{0}\right)\right|\left|a^{\alpha} e^{i \pi \alpha / 2}+\frac{k \alpha}{a^{\alpha}} e^{i \pi(1-\alpha) / 2}\right|
$$




$$
\begin{aligned}
& =\left|p\left(z_{0}\right)\right| \sqrt{\left(\frac{k \alpha}{a^{\alpha}}\right)^{2}+\left(a^{\alpha}\right)^{2}+2 k \alpha \cos \left(\frac{\pi}{2}(1-2 \alpha)\right)} \\
& \geqq\left|p\left(z_{0}\right)\right| \sqrt{\left(\frac{\alpha}{2}\left(a^{1-\alpha}+a^{-1-\alpha}\right)\right)^{2}+0+2 \alpha \cos \left(\frac{\pi}{2}(1-2 \alpha)\right)} .
\end{aligned}
$$

We now define a real function $g$ by

$$
g(a)=a^{1-\alpha}+a^{-1-\alpha} \quad(a>0) .
$$

Then this function $g$ takes the minimum value for $a$ given by

$$
a=\sqrt{\frac{1+\alpha}{1-\alpha}}
$$

Therefore, from the above equality in the case when $0<\alpha<1$, we obtain

$$
\left|\left[p\left(z_{0}\right)\right]^{2}+\frac{z p^{\prime}\left(z_{0}\right)}{p\left(z_{0}\right)}\right| \geqq\left|p\left(z_{0}\right)\right| \sqrt{\frac{\alpha^{2}}{4}\left[\left(\frac{1+\alpha}{1-\alpha}\right)^{(1-\alpha) / 2}+\left(\frac{1+\alpha}{1-\alpha}\right)^{(-1-\alpha) / 2}\right]^{2}+2 \alpha \sin (\pi \alpha)},
$$

which contradicts our hypothesis of Theorem 2.1 .

For the case when

$$
\left[p\left(z_{0}\right)\right]^{\frac{1}{\alpha}}=-i a \quad(a>0)
$$

by utilizing the same method as above, Lemma 1.1 for $\alpha=1$ yields

$$
\begin{aligned}
\left.\mid\left[p^{(} z_{0}\right)\right]^{2}+\frac{z p^{\prime}\left(z_{0}\right)}{p\left(z_{0}\right)} \mid & =\left|p\left(z_{0}\right)\right|\left|a^{\alpha} e^{-i \pi \alpha / 2}+\frac{k \alpha}{a^{\alpha}} e^{i \pi(1+\alpha) / 2}\right| \\
& =\left|p\left(z_{0}\right)\right|\left|a e^{-i \pi / 2}-\frac{k}{a}\right| \\
& =\left|p\left(z_{0}\right)\right|\left|-i a-\frac{k}{a}\right|=\left|p\left(z_{0}\right)\right| \sqrt{a^{2}+\left(\frac{k}{a}\right)^{2}} \\
& \geqq\left|p\left(z_{0}\right)\right| \sqrt{\frac{1}{4}}=\frac{1}{2}\left|p\left(z_{0}\right)\right| .
\end{aligned}
$$

Also, for $0<\alpha<1$, it follows for $k \leqq-1$ that

$$
\begin{aligned}
& \left|\left[p\left(z_{0}\right)\right]^{2}+\frac{z p^{\prime}\left(z_{0}\right)}{p\left(z_{0}\right)}\right| \\
& \quad=\left|p\left(z_{0}\right)\right|\left|a^{\alpha} e^{-i \pi \alpha / 2}+\frac{k \alpha}{a^{\alpha}} e^{i \pi(1+\alpha) / 2}\right| \\
& \quad=\left|p\left(z_{0}\right)\right| \sqrt{\left(\frac{k}{a}\right)^{2}+\left(a^{\alpha}\right)^{2}+2 k \alpha \cos \left(\frac{\pi}{2}(1+2 \alpha)\right)} \\
& \quad \geqq\left|p\left(z_{0}\right)\right| \sqrt{\frac{\alpha^{2}}{4}\left[\left(\frac{1+\alpha}{1-\alpha}\right)^{(1-\alpha) / 2}+\left(\frac{1+\alpha}{1-\alpha}\right)^{(-1-\alpha) / 2}\right]^{2}+2 k \alpha \cos \left(\frac{\pi}{2}(1+2 \alpha)\right)}
\end{aligned}
$$




$$
\geqq\left|p\left(z_{0}\right)\right| \sqrt{\frac{\alpha^{2}}{4}\left[\left(\frac{1+\alpha}{1-\alpha}\right)^{(1-\alpha) / 2}+\left(\frac{1+\alpha}{1-\alpha}\right)^{(-1-\alpha) / 2}\right]^{2}+2 \alpha \sin (\pi \alpha)},
$$

which also contradicts our hypothesis of Theorem 2.1. From the two above-discussed contradictions, it follows that

$$
|\arg (p(z))|<\frac{\alpha \pi}{2} \quad(\forall z \in \mathbb{U}) .
$$

This completes the proof of Theorem 2.1.

Remark 2.1 If

$$
f \in \mathcal{A} \text { and } \quad p(z):=\frac{z f^{\prime}(z)}{f(z)} \neq 0,
$$

then $p^{\prime}(0) \neq 0$ is equivalent to $f^{\prime \prime}(0) \neq 0$ and Theorem 2.1 leads to the following result, which gives a sufficient condition for strong starlikeness of order $\alpha$.

Corollary 2.1 Let the function $f \in \mathcal{A}$, with $f^{\prime \prime}(0) \neq 0$, satisfy the following inequality:

$$
\left|1+\frac{z f^{\prime \prime}(z)}{f^{\prime}(z)}+\frac{z f^{\prime}(z)}{f(z)}\left(\frac{z f^{\prime}(z)}{f(z)}-1\right)\right|<A(\alpha)\left|\frac{z f^{\prime}(z)}{f(z)}\right|
$$

where $A(\alpha)$ is given by (2.2). Then $f \in \widetilde{\mathcal{S}}^{*}(\alpha)$.

Remark 2.2 For $f \in \mathcal{A}, \alpha=1$ and $p(z):=f^{\prime}(z) \neq 0$, Theorem 2.1 leads to the following result which gives a sufficient condition for the close-to-convexity (univalence) of the function $f$.

Corollary 2.2 If the function $f \in \mathcal{A}$, with $f^{\prime \prime}(0) \neq 0$, satisfies the following inequality:

$$
\left|\left[f^{\prime}(z)\right]^{2}+\frac{z f^{\prime}(z)}{f(z)}\right|<\frac{1}{2}\left|f^{\prime}(z)\right|
$$

then $f \in \mathcal{C}$.

We now state and prove the following result.

Theorem 2.2 Let $p$ be an analytic function in $\mathbb{U}$, with $p(0)=1, p^{\prime}(0) \neq 0$, and $p(z) \neq 0$ for $z \in \mathbb{U}$, that satisfies the following inequality:

$$
\left|p(z)+\frac{z p^{\prime}(z)}{[p(z)]^{2}}\right|<B(\alpha)|p(z)|
$$

where

$$
B(\alpha)=\left\{\begin{array}{l}
\sqrt{1+\frac{\alpha^{2}}{4}\left(\widetilde{\alpha}^{(1-2 \alpha) / 2}+\widetilde{\alpha}^{(-1-2 \alpha) / 2}\right)^{2}+\alpha\left(\widetilde{\alpha}^{(1-2 \alpha) / 2}+\widetilde{\alpha}^{(-1-2 \alpha) / 2}\right) \sin (\pi \alpha)} \\
\text { if } 0<\alpha<\frac{1}{2}, \\
\sqrt{\frac{5}{4}} \quad \text { if } \alpha=\frac{1}{2}
\end{array}\right.
$$


and

$$
\widetilde{\alpha}=\frac{1+2 \alpha}{1-2 \alpha}
$$

Then $p \in \widetilde{\mathcal{P}}(\alpha)$.

Proof If we suppose that there exists a point $z_{0} \in \mathbb{U}$ such that

$$
|\arg (p(z))|<\frac{\alpha \pi}{2} \quad \text { for }|z|<\left|z_{0}\right|
$$

and

$$
\left|\arg \left(p\left(z_{0}\right)\right)\right|=\frac{\alpha \pi}{2}
$$

we find from Lemma 1.1 that

$$
\frac{z p^{\prime}\left(z_{0}\right)}{p\left(z_{0}\right)}=i k \alpha
$$

where

$$
\left[p\left(z_{0}\right)\right]^{\frac{1}{\alpha}}= \pm i a \quad(a>0)
$$

and $k$ is given by (1.2) or (1.3) for $m=1$.

For the case when

$$
p\left(z_{0}\right)^{\frac{1}{\alpha}}=i a \quad(a>0)
$$

we have

$$
\begin{aligned}
\left|p\left(z_{0}\right)+\frac{z p^{\prime}\left(z_{0}\right)}{\left[p\left(z_{0}\right)\right]^{2}}\right| & =\left|p\left(z_{0}\right)\right| \cdot\left|1+\frac{z p^{\prime}\left(z_{0}\right)}{p\left(z_{0}\right)} \frac{1}{\left[p\left(z_{0}\right)\right]^{2}}\right|=\left|p\left(z_{0}\right)\right| \cdot\left|1+i k \alpha \frac{1}{(i a)^{2 \alpha}}\right| \\
& =\left|p\left(z_{0}\right)\right| \cdot\left|1+\frac{k \alpha}{a^{2 \alpha}} e^{i \pi(1-2 \alpha) / 2}\right| .
\end{aligned}
$$

Now, from (2.5) for $\alpha=\frac{1}{2}$, we get

$$
\begin{aligned}
\left|p\left(z_{0}\right)+\frac{z p^{\prime}\left(z_{0}\right)}{\left[p\left(z_{0}\right)\right]^{2}}\right| & =\left|p\left(z_{0}\right)\right|\left|1-i \frac{k}{a}\right|=\left|p\left(z_{0}\right)\right| \sqrt{1+\left(\frac{k}{a}\right)^{2}} \\
& \geqq \sqrt{\frac{5}{4}}\left|p\left(z_{0}\right)\right| .
\end{aligned}
$$

Also, from (2.5) for $0<\alpha<\frac{1}{2}$, we deduce that

$$
\begin{aligned}
& \left|p\left(z_{0}\right)+\frac{z p^{\prime}\left(z_{0}\right)}{\left[p\left(z_{0}\right)\right]^{2}}\right| \\
& \quad=\left|p\left(z_{0}\right)\right|\left|1+\frac{k \alpha}{a^{2 \alpha}} e^{i \pi(1-2 \alpha) / 2}\right|
\end{aligned}
$$




$$
\begin{aligned}
& =\left|p\left(z_{0}\right)\right| \sqrt{1+\left(\frac{k \alpha}{a^{2 \alpha}}\right)^{2}+2 \frac{k \alpha}{a^{2 \alpha}} \cos \left(\frac{\pi}{2}(1-2 \alpha)\right)} \\
& \geqq\left|p\left(z_{0}\right)\right| \sqrt{1+\left(\frac{\alpha}{2}\left(a^{1-2 \alpha}+a^{-1-2 \alpha}\right)\right)^{2}+\alpha\left(a^{1-2 \alpha}+a^{-1-2 \alpha}\right) \cos \left(\frac{\pi}{2}(1-2 \alpha)\right)} .
\end{aligned}
$$

We now define a real function $h$ by

$$
h(a)=a^{1-2 \alpha}+a^{-1-2 \alpha} \quad(a>0) .
$$

Then this function takes the minimum value for $a$ given by

$$
a=\sqrt{\frac{1+2 \alpha}{1-2 \alpha}}
$$

Therefore, from the above equality, when

$$
0<\alpha<\frac{1}{2} \quad \text { for } \tilde{\alpha}=\frac{1+2 \alpha}{1-2 \alpha}
$$

we obtain

$$
\begin{aligned}
& \left|p\left(z_{0}\right)+\frac{z p^{\prime}\left(z_{0}\right)}{\left[p\left(z_{0}\right)\right]^{2}}\right| \\
& \geqq\left|p\left(z_{0}\right)\right| \\
& \quad \times \sqrt{1+\frac{\alpha^{2}}{4}\left(\widetilde{\alpha}^{(1-2 \alpha) / 2}+\widetilde{\alpha}^{(-1-2 \alpha) / 2}\right)^{2}+\alpha\left(\widetilde{\alpha}^{(1-2 \alpha) / 2}+\widetilde{\alpha}^{(-1-2 \alpha) / 2}\right) \cos \left(\frac{\pi}{2}(1-2 \alpha)\right)},
\end{aligned}
$$

which contradicts our hypothesis in Theorem 2.2.

Next, for the case when

$$
\left[p\left(z_{0}\right)\right]^{\frac{1}{\alpha}}=-i a \quad(a>0)
$$

using the same method as before, we can obtain a contradiction to the assumption in Theorem 2.2.

From the two above-discussed contradictions, it follows that

$$
|\arg (p(z))|<\frac{\alpha \pi}{2} \quad(\forall z \in \mathbb{U}) .
$$

This completes the proof of Theorem 2.2.

Corollary 2.3 Let the function $f \in \mathcal{A}$, with $f^{\prime \prime}(0) \neq 0$, satisfy the following inequality:

$$
\left|\frac{f(z)}{z f^{\prime}(z)}\left(1+\frac{z f^{\prime \prime}(z)}{f^{\prime}(z)}\right)+\frac{z f^{\prime}(z)}{f(z)}-1\right|<B(\alpha)\left|\frac{z f^{\prime}(z)}{f(z)}\right|
$$

where $B(\alpha)$ is given by (2.4). Then $f \in \widetilde{\mathcal{S}}^{*}(\alpha)$. 
Theorem 2.3 Let $p$ be an analytic function in $\mathbb{U}$, with $p(0)=1, p^{\prime}(0) \neq 0$, and $p(z) \neq 0$ for $z \in \mathbb{U}$, that satisfies the following inequality:

$$
\left|\arg \left(p(z)+\frac{z p^{\prime}(z)}{[p(z)]^{2}}\right)\right|<\frac{\delta \pi}{2}
$$

where

$$
\delta=\alpha+\frac{2}{\pi} \arctan \left(\frac{\frac{\alpha}{2}\left(\widetilde{\alpha}^{(1-2 \alpha) / 2}+\widetilde{\alpha}^{(-1-2 \alpha) / 2}\right) \cos (\pi \alpha)}{1+\frac{\alpha}{2}\left(\widetilde{\alpha}^{(1-2 \alpha) / 2}+\widetilde{\alpha}^{(-1-2 \alpha) / 2}\right) \sin (\pi \alpha)}\right)
$$

and

$$
\widetilde{\alpha}=\frac{1+2 \alpha}{1-2 \alpha} \quad\left(0<\alpha<\frac{1}{2}\right)
$$

Then $p \in \widetilde{\mathcal{P}}(\alpha)$.

Proof Using similar arguments as in the proof of Theorem 2.1, for the case when

$$
\left[p\left(z_{0}\right)\right]^{\frac{1}{\alpha}}=i a \quad(a>0)
$$

we have

$$
\begin{aligned}
\arg \left(p\left(z_{0}\right)+\frac{z p^{\prime}\left(z_{0}\right)}{\left[p\left(z_{0}\right)\right]^{2}}\right) & =\arg \left(p\left(z_{0}\right)\left(1+\frac{z p^{\prime}\left(z_{0}\right)}{p\left(z_{0}\right)} \frac{1}{\left[p\left(z_{0}\right)\right]^{2}}\right)\right) \\
& =\arg \left(p\left(z_{0}\right)\right)+\arg \left(1+i k \alpha \frac{1}{(i a)^{2 \alpha}}\right) \\
& =\arg \left(p\left(z_{0}\right)\right)+\arg \left(1+\frac{k \alpha}{a^{2 \alpha}} e^{i \pi(1-2 \alpha) / 2}\right) .
\end{aligned}
$$

Since

$$
\frac{k \alpha}{a^{2 \alpha}} \geqq \frac{\alpha}{2}\left(a^{1-2 \alpha}+a^{-1-2 \alpha}\right),
$$

we now define a real function $h$ by

$$
h(a)=a^{1-2 \alpha}+a^{-1-2 \alpha} \quad(a>0) .
$$

Then this function takes on the minimum value for $a$ given by

$$
a=\sqrt{\frac{1+2 \alpha}{1-2 \alpha}}
$$

Therefore, from the above inequality, when

$$
0<\alpha<\frac{1}{2} \quad \text { for } \tilde{\alpha}=\frac{1+2 \alpha}{1-2 \alpha}
$$


we obtain

$$
\frac{k \alpha}{a^{2 \alpha}} \geqq \frac{\alpha}{2}\left(\widetilde{\alpha}^{(1-2 \alpha) / 2}+\widetilde{\alpha}^{(-1-2 \alpha) / 2}\right) .
$$

Therefore

$$
\begin{aligned}
\arg \left(p\left(z_{0}\right)+\frac{z p^{\prime}\left(z_{0}\right)}{\left[p\left(z_{0}\right)\right]^{2}}\right) & \geqq \frac{\alpha \pi}{2}+\arctan \left(\frac{\frac{\alpha}{2}\left(\widetilde{\alpha}^{(1-2 \alpha) / 2}+\widetilde{\alpha}^{(-1-2 \alpha) / 2}\right) \cos (\pi \alpha)}{1+\frac{\alpha}{2}\left(\widetilde{\alpha}^{(1-2 \alpha) / 2}+\widetilde{\alpha}^{(-1-2 \alpha) / 2}\right) \sin (\pi \alpha)}\right) \\
& =\frac{\delta \pi}{2}
\end{aligned}
$$

which contradicts our hypothesis in Theorem 2.3.

Next, for the case when

$$
\left[p\left(z_{0}\right)\right]^{\frac{1}{\alpha}}=-i a \quad(a>0),
$$

with

$$
\frac{k \alpha}{a^{2 \alpha}} \leqq-\frac{\alpha}{2}\left(a^{1-2 \alpha}+a^{-1-2 \alpha}\right),
$$

using the same method as before, we can obtain

$$
\begin{aligned}
\arg \left(p\left(z_{0}\right)+\frac{z p^{\prime}\left(z_{0}\right)}{\left[p\left(z_{0}\right)\right]^{2}}\right) & =\arg \left(p\left(z_{0}\right)\right)+\arg \left(1+i k \alpha \frac{1}{(-i a)^{2 \alpha}}\right) \\
& =\arg \left(p\left(z_{0}\right)\right)+\arg \left(1+\frac{k \alpha}{a^{2 \alpha}} e^{i \pi(1+2 \alpha) / 2}\right) \\
& =-\frac{\alpha \pi}{2}+\arctan \left(\frac{\frac{k \alpha}{a^{2 \alpha}} \sin \left(\frac{\pi}{2}(1+2 \alpha)\right)}{1+\frac{k \alpha}{a^{2 \alpha}} \cos \left(\frac{\pi}{2}(1+2 \alpha)\right)}\right) \\
& =-\frac{\alpha \pi}{2}+\arctan \left(\frac{\frac{k \alpha}{a^{2 \alpha}} \cos (\pi \alpha)}{1-\frac{k \alpha}{a^{2 \alpha}} \sin (\pi \alpha)}\right) \\
& \leqq-\frac{\alpha \pi}{2}-\arctan \left(\frac{\frac{\alpha}{2}\left(\widetilde{\alpha}^{(1-2 \alpha) / 2}+\widetilde{\alpha}^{(-1-2 \alpha) / 2}\right) \cos (\pi \alpha)}{1+\frac{\alpha}{2}\left(\widetilde{\alpha}^{(1-2 \alpha) / 2}+\widetilde{\alpha}^{(-1-2 \alpha) / 2}\right) \sin (\pi \alpha)}\right) \\
& =-\frac{\delta \pi}{2},
\end{aligned}
$$

which is a contradiction to the assumption of Theorem 2.3.

From the two above-discussed contradictions, it follows that

$$
|\arg (p(z))|<\frac{\alpha \pi}{2} \quad(\forall z \in \mathbb{U}) .
$$

This completes the proof of Theorem 2.3.

Corollary 2.4 Let the function $f \in \mathcal{A}$, with $f^{\prime \prime}(0) \neq 0$, satisfy the following inequality:

$$
\left|\arg \left(\frac{f(z)}{z f^{\prime}(z)}\left(1+\frac{z f^{\prime \prime}(z)}{f^{\prime}(z)}\right)+\frac{z f^{\prime}(z)}{f(z)}-1\right)\right|<\frac{\delta \pi}{2},
$$

where $\delta$ is given by (2.6). Then $f \in \widetilde{\mathcal{S}}^{*}(\alpha)$. 
Theorem 2.4 Let $p$ be an analytic function in $\mathbb{U}$, with $p(0)=1, p^{\prime}(0) \neq 0$, and $p(z) \neq 0$ for $z \in \mathbb{U}$, that satisfies the following inequality:

$$
\left|\arg \left([p(z)]^{2}+\frac{z p^{\prime}(z)}{p(z)}\right)\right|<\frac{\gamma \pi}{2}
$$

where

$$
\gamma=2 \alpha+\frac{2}{\pi} \arctan \left(\frac{\frac{\alpha}{2}\left(\widetilde{\alpha}^{(1-2 \alpha) / 2}+\widetilde{\alpha}^{(-1-2 \alpha) / 2}\right) \cos (\pi \alpha)}{1+\frac{\alpha}{2}\left(\widetilde{\alpha}^{(1-2 \alpha) / 2}+\widetilde{\alpha}^{(-1-2 \alpha) / 2}\right) \sin (\pi \alpha)}\right)
$$

and

$$
\widetilde{\alpha}=\frac{1+2 \alpha}{1-2 \alpha} \quad\left(0<\alpha<\frac{1}{2}\right) .
$$

Then $p \in \widetilde{\mathcal{P}}(\alpha)$.

Proof By using a similar method as in the proof of Theorem 2.1, for the case when

$$
\left[p\left(z_{0}\right)\right]^{\frac{1}{\alpha}}=i a \quad(a>0)
$$

with

$$
\frac{k \alpha}{a^{2 \alpha}} \geqq \frac{\alpha}{2}\left(a^{1-2 \alpha}+a^{-1-2 \alpha}\right)
$$

we have

$$
\begin{aligned}
\arg \left(\left[p\left(z_{0}\right)\right]^{2}+\frac{z_{0} p^{\prime}\left(z_{0}\right)}{p\left(z_{0}\right)}\right) & =\arg \left(\left[p\left(z_{0}\right)\right]^{2}\left(1+\frac{z p^{\prime}\left(z_{0}\right)}{p\left(z_{0}\right)} \frac{1}{\left[p\left(z_{0}\right)\right]^{2}}\right)\right) \\
& =\arg \left(\left[p\left(z_{0}\right)\right]^{2}\right)+\arg \left(1+i k \alpha \frac{1}{(i a)^{2 \alpha}}\right) \\
& =2 \arg \left(p\left(z_{0}\right)\right)+\arg \left(1+\frac{k \alpha}{a^{2 \alpha}} e^{i \pi(1-2 \alpha) / 2}\right) \\
& =\alpha \pi+\arctan \left(\frac{\frac{k \alpha}{a^{2 \alpha}} \sin \left(\frac{\pi}{2}(1-2 \alpha)\right)}{1+\frac{k \alpha}{a^{2 \alpha}} \cos \left(\frac{\pi}{2}(1-2 \alpha)\right)}\right) .
\end{aligned}
$$

We now define a real function $h$ by

$$
h(a)=a^{1-2 \alpha}+a^{-1-2 \alpha} \quad(a>0) .
$$

Then this function takes on the minimum value for $a$ given by

$$
a=\sqrt{\frac{1+2 \alpha}{1-2 \alpha}}
$$

Therefore, from the above equality, when

$$
0<\alpha<\frac{1}{2} \text { for } \widetilde{\alpha}=\frac{1+2 \alpha}{1-2 \alpha}
$$


we obtain

$$
\begin{aligned}
\arg \left(\left[p\left(z_{0}\right)\right]^{2}+\frac{z p^{\prime}\left(z_{0}\right)}{p\left(z_{0}\right)}\right) & \geqq \alpha \pi+\arctan \left(\frac{\frac{\alpha}{2}\left(\widetilde{\alpha}^{(1-2 \alpha) / 2}+\widetilde{\alpha}^{(-1-2 \alpha) / 2}\right) \cos (\pi \alpha)}{1+\frac{\alpha}{2}\left(\widetilde{\alpha}^{(1-2 \alpha) / 2}+\widetilde{\alpha}^{(-1-2 \alpha) / 2}\right) \sin (\pi \alpha)}\right) \\
& =\frac{\gamma \pi}{2},
\end{aligned}
$$

which contradicts our hypothesis in Theorem 2.4.

For the case when

$$
\left[p\left(z_{0}\right)\right]^{\frac{1}{\alpha}}=-i a \quad(a>0)
$$

by using the same method as before, we can obtain

$$
\arg \left(\left[p\left(z_{0}\right)\right]^{2}+\frac{z p^{\prime}\left(z_{0}\right)}{p\left(z_{0}\right)}\right) \leqq-\frac{\gamma \pi}{2}
$$

which is a contradiction to the assumption in Theorem 2.4.

From the two above-discussed contradictions, it follows that

$$
|\arg (p(z))|<\frac{\alpha \pi}{2} \quad(\forall z \in \mathbb{U}) .
$$

This completes the proof of Theorem 2.4.

Corollary 2.5 Suppose that the function $f \in \mathcal{A}$, with $f^{\prime \prime}(0) \neq 0$, satisfies the following inequality:

$$
\left|\arg \left(1+\frac{z f^{\prime \prime}(z)}{f^{\prime}(z)}+\frac{z f^{\prime}(z)}{f(z)}\left(\frac{z f^{\prime}(z)}{f(z)}-1\right)\right)\right|<\frac{\gamma \pi}{2},
$$

where $\gamma$ is given by (2.7). Then $f \in \widetilde{\mathcal{S}}^{*}(\alpha)$.

Remark 2.3 For $g \in \mathcal{S}^{*}$ and $f \in \mathcal{A}$ such that $2 f^{\prime \prime}(0) \neq g^{\prime \prime}(0)$, by setting

$$
p(z):=\frac{z f^{\prime}(z)}{g(z)} \neq 0
$$

in the above theorems, we will obtain a sufficient condition for strong close-to-convexity.

\section{Conclusion}

In the present paper, we have derived some sufficient conditions (or criteria) for the Carathéodory functions as a certain class of analytic functions in the open unit disk $\mathbb{U}$. We have also deduced various sufficient conditions for the univalence, strong starlikeness, and strong close-to-convexity of functions in the normalized analytic function class $\mathcal{A}$. We have considered several other related results as well. Also, with a view to motivating further research on the subject-matter of this investigation, we have included the citations of other closely-related recent developments as well. 


\section{Acknowledgements}

The first author was supported by the Basic Science Research Program through the National Research Foundation of Korea (NRF) funded by the Ministry of Education, Science and Technology (No. 2019R111A3A01050861).

\section{Funding}

Not applicable.

\section{Availability of data and materials}

Not applicable.

\section{Competing interests}

The authors declare that they have no competing interests.

\section{Authors' contributions}

All authors jointly worked on the results and they read and approved the final manuscript.

\section{Author details}

'Department of Applied Mathematics, College of Natural Sciences, Pukyong National University, Busan, Republic of Korea. ${ }^{2}$ Department of Mathematics and Statistics, University of Victoria, Victoria, Canada. ${ }^{3}$ Department of Medical Research, China Medical University Hospital, China Medical University, Taichung, Taiwan. ${ }^{4}$ Department of Mathematics and Informatics, Azerbaijan University, Baku, Azerbaijan. ${ }^{5}$ Faculty of Mathematical Sciences, Shahrood University of Technology, Shahrood, Iran.

\section{Publisher's Note}

Springer Nature remains neutral with regard to jurisdictional claims in published maps and institutional affiliations.

Received: 28 September 2019 Accepted: 18 March 2020 Published online: 30 March 2020

\section{References}

1. Ali, R.M., Cho, N.E., Ravichandran, V., Kumar, S.S.: Differential subordination for functions associated with the lemniscate of Bernoulli. Taiwan. J. Math. 16, 1017-1026 (2012)

2. Analouei Adegani, E., Bulboacă, T., Motamednezhad, A.: Simple sufficient subordination conditions for close-to-convexity. Mathematics 7, 241 (2019)

3. Analouei Adegani, E., Cho, N.E., Jafari, M.: Logarithmic coefficients for univalent functions defined by subordination. Mathematics 7, 408 (2019)

4. Aouf, M.K., Bulboaca, T.: Subordination and superordination properties of multivalent functions defined by certain integral operator. J. Franklin Inst. 347, 641-653 (2010)

5. Aouf, M.K., Hossen, H.M., Lashin, A.Y.: An application of certain integral operators. J. Math. Anal. Appl. 248, 475-481 (2000)

6. Aouf, M.K., Hossen, H.M., Srivastava, H.M.: Some families of multivalent functions. Comput. Math. Appl. 39, 39-48 (2000)

7. Bulboacă, T:: On some classes of differential subordinations. Stud. Univ. Babeş-Bolyai, Math. 31, 45-50 (1986)

8. Carathéodory, C.: Über den variabilitätsbereich der coeffizienten von potenzreihen, die gegebene werte nicht annehmen. Math. Ann. 64(1), 95-115 (1907)

9. Carathéodory, C.: Über den variabilitätsbereich der Fourier'schen konstanten von positiven harmonischen Funktionen. Rend. Circ. Mat. Palermo 32, 193-217 (1911)

10. Cho, N.E., Kumar, S., Kumar, V., Ravichandran, V.: Differential subordination and radius estimates for starlike functions associated with the Booth lemniscate. Turk. J. Math. 42(3), 1380-1399 (2018)

11. Cho, N.E., Kumar, S., Kumar, V., Ravichandran, V., Srivastava, H.M.: Starlike functions related to the Bell numbers. Symmetry 11(2), 219 (2019)

12. Kim, I.H., Sim, Y.J., Cho, N.E.: New criteria for Carathéodory functions. J. Inequal. Appl. 2019(1), 13 (2019)

13. Kumar, S., Ravichandran, V:: Subordinations for functions with positive real part. Complex Anal. Oper. Theory 12 1179-1191 (2018)

14. Miller, S.S., Mocanu, P.T.: Second-order differential inequalities in the complex plane. J. Math. Anal. Appl. 65, 298-305 (1978)

15. Nunokawa, M.: On properties of non-Carathéodory functions. Proc. Jpn. Acad., Ser. A, Math. Sci. 68, 152-153 (1992)

16. Nunokawa, M.: On the order of strongly starlikeness of strongly convex functions. Proc. Jpn. Acad., Ser. A, Math. Sci. 69, 234-237 (1993)

17. Nunokawa, M., Sokół, J: On some differential subordinations. Studia Sci. Math. Hung. 54, 436-445 (2017)

18. Nunokawa, M., Sokół, J.: On multivalent starlike functions and Ozaki condition. Complex Var. Elliptic Equ. 64(1), 78-92 (2019)

19. Shiraishi, H., Owa, S., Srivastava, H.M.: Sufficient conditions for strongly Carathéodory functions. Comput. Math. Appl. 62, 2978-2987 (2011)

20. Sim, Y.., Kwon, O.S., Cho, N.E., Srivastava, H.M.: Some sets of sufficient conditions for Carathéodory functions. J. Comput. Anal. Appl. 21, 1243-1254 (2016)

21. Srivastava, H.M., Aouf, M.K., Mostafa, A.O., Zayed, H.M.: Certain subordination-preserving family of integral operators associated with p-valent functions. Appl. Math. Inf. Sci. 11, 951-960 (2017)

22. Srivastava, H.M., Hussain, S., Raziq, A., Raza, M.: The Fekete-Szegö functional for a subclass of analytic functions associated with quasi-subordination. Carpath. J. Math. 34, 103-113 (2018)

23. Srivastava, H.M., Owa, S.: Some new results associated with Carathéodory functions of order $\alpha$. J. Comput. Sci. Appl. Math. 2(1), 11-13 (2016) 
24. Srivastava, H.M., Prajapati, A., Gochhayat, P.: Third-order differential subordination and differential superordination results for analytic functions involving the Srivastava-Attiya operator. Appl. Math. Inf. Sci. 12, 469-481 (2018)

25. Srivastava, H.M., Răducanu, D., Zaprawa, P.: A certain subclass of analytic functions defined by means of differential subordination. Filomat 30, 3743-3757 (2016)

26. Tang, H., Srivastava, H.M., Deng, G.-T.: Some families of analytic functions in the upper half-plane and their associated differential subordination and differential superordination properties and problems. Appl. Math. Inf. Sci. 11 $1247-1257(2017)$

27. Xu, Q.-H., Xiao, H.-G., Srivastava, H.M.: Some applications of differential subordination and the Dziok-Srivastava convolution operator. Appl. Math. Comput. 230, 496-508 (2014)

28. Xu, Q.-H., Yang, T., Srivastava, H.M.: Sufficient conditions for a general class of Carathéodory functions. Filomat 30, 3615-3625 (2016)

Submit your manuscript to a SpringerOpen ${ }^{\circ}$ journal and benefit from:

- Convenient online submission

- Rigorous peer review

- Open access: articles freely available online

- High visibility within the field

- Retaining the copyright to your article

Submit your next manuscript at $\gg$ springeropen.com 